\title{
Knockdown of connexin 43 attenuates balloon injury-induced vascular restenosis through the inhibition of the proliferation and migration of vascular smooth muscle cells
}

\author{
XIAO-JIAN HAN ${ }^{1,3,7}$, DAN HE ${ }^{1,4}$, LIANG-JING XU ${ }^{5}$, MIN CHEN $^{1,6}$, YI-QI WANG ${ }^{1}$, JIU-GENG FENG ${ }^{2}$, \\ MIN-JUN WEI ${ }^{2}$, TAO HONG ${ }^{2}$ and LI-PING JIANG ${ }^{1}$
}

${ }^{1}$ Department of Pharmacology, School of Pharmaceutical Science, ${ }^{2}$ Department of Neurosurgery, The First Affiliated Hospital,
and ${ }^{3}$ Institute of Translational Medicine, Nanchang University, Nanchang, Jiangxi 330006; ${ }^{4}$ Department of Pharmacy,
Nanchang Municipal Liver Diseases Hospital, Nanchang, Jiangxi 330002; ${ }^{5}$ Department of Hematology,
The First Affiliated Hospital of Soochow University, Suzhou, Jiangsu 215000; ${ }^{6}$ Department of Pharmacy,
Zhongshan Hospital of Hubei, Wuhan, Hubei 430033, P.R. China

Received February 3, 2015; Accepted August 24, 2015

DOI: $10.3892 /$ ijmm.2015.2346

\begin{abstract}
Coronary artery disease (CAD) or atherosclerotic heart disease is one of the most common types of cardiovascular disease. Although percutaneous coronary intervention [PCI or percutaneous transluminal coronary angioplasty (PTCA)] is a mature, well-established technique used to treat atherosclerotic heart disease, its long-term therapeutic effects are compromised by a high incidence of vascular restenosis (RS) following angioplasty. In our previous study, we found that the principal gap junction protein, connexin 43 (Cx43), in vascular smooth muscle cells (VSMCs) was involved in the development of vascular RS following angioplasty-induced balloon injury. However, the exact role action of $\mathrm{Cx} 43$ in vascular RS remains unclear. In the present study, we aimed to further examine whether the knockdown of $\mathrm{Cx} 43$ attenuates the development of vascular RS through the inhibition of the proliferation and migration of VSMCs. We found that the use of a lentiviral
\end{abstract}

Correspondence to: Professor Li-Ping Jiang, Department of Pharmacology, School of Pharmaceutical Science, Nanchang University, 603 Bayi Road, Nanchang, Jiangxi 330006, P.R. China E-mail: lipingjiang2002@aliyun.com

Professor Tao Hong, Department of Neurosurgery, The First Affiliated Hospital, Nanchang University, 17 Yong Wai Street, Nanchang, Jiangxi 330006, P.R. China

E-mail: ht2000@vip.sina.com

Present address: ${ }^{7}$ Affiliated Eye Hospital of Nanchang University, Nanchang, Jiangxi 330006, P.R. China

Abbreviations: RS, restenosis; PCI, percutaneous coronary intervention; VSMCs, vascular smooth muscle cells; GJ, gap junctions; $\mathrm{Cx} 43$, connexin 43; RNAi, RNA interference; CAD, coronary artery disease

Key words: connexin 43, vascular smooth muscle cells, proliferation, migration, vascular restenosis vector expressing shRNA targeting Cx43 (Cx43-RNAi-LV) efficiently silenced the mRNA and protein expression of Cx43 in cultured VSMCs. In addition, MTT and Transwell assays were used to examined the proliferation and migration of the VSMCs, respectively. The results revealed that the knockdown of Cx43 by Cx43-RNAi-LV at a multiplicity of infection (MOI) of 100 significantly inhibited the proliferation and migration of the VSMCs in vitro. Notably, the knockdown of Cx43 also effectively attenuated the development of vascular RS and intimal hyperplasia following balloon injury in vivo. Taken together, our data suggest that $\mathrm{Cx} 43$ is involved in the development of vascular RS and intimal hyperplasia through the regulation of the proliferation and migration of VSMCs. Thus, the present study provides new insight into the pathogenesis of vascular RS, and suggests that further comfirms that $\mathrm{Cx} 43$ may well be a novel potential pharmacological target for preventing vascular RS following PCI.

\section{Introduction}

Coronary artery disease (CAD) or atherosclerotic heart disease is one of the most common types of cardiovascular disease with a high incidence of morbidity and mortality. It is well acknowledged that atherosclerosis is the main cause of CAD. Of the therapeutic measures currently available, percutaneous coronary intervention [PCI or percutaneous transluminal coronary angioplasty (PTCA)] has developed into a mature, well-established technique used to treat CAD since it was first introduced in 1977 (1). PCI effectively improves coronary blood flow, reduces angina pectoris and greatly improves the quality of life of patients (2). Another advantage of PCI is that it is a relatively easy technique and is minimally invasive. However, its long-term therapeutic effects are often compromised by the high incidence of vascular restenosis (RS) following angioplasty. In clinical practice, the incidence of RS is approximately 20-50\% at 6 months post-PCI and even with stent implantation, the incidence of RS is also approximately $25-30 \%$ (3). Therefore, vascular RS is a serious complication of PCI, and it is neces- 
sary to fully elucidate the molecular mechanisms responsible for the development of RS following angioplasty so that a novel therapeutic methods can be found to prevent the occurrence of vascular RS following angioplasty.

It has been suggested that the proliferation and migration of vascular smooth muscle cells (VSMCs) may be key factors involved in the development of RS following angioplasty (4). Mature VSMCs are highly differentiated, and they are principally responsible for contraction. However, VSMCs also proliferate and produce the matrix components of the blood vessel wall under specific pathophysiological conditions, such as vasculogenesis (4). Moreover, VSMCs still retain remarkable plasticity, and may undergo relatively rapid and reversible changes in their phenotype in response to local environmental stress (5). Vascular RS at an early stage, often occurs several hours or several days following PCI, and RS is mainly induced by vasospasm or decreased vessel elasticity. Subsequently, some cytokines, including endothelin, angiotensin II, basic fibroblast growth factor, platelet-derived growth factor and transforming growth factor are released from damaged endothelial cells and macrophages which have invaded the endothelial sublayer. These cytokines stimulate the migration and proliferation of VSMCs, and induce the accumulation of extracellular matrix components, which leads to the remodeling of the blood vessel wall and ultimately to vascular RS (6). Although some intracellular signaling pathways have been found to regulate the proliferation and migration of VSMCs $(2,7,8)$, the role of direct intercellular communication pathways and gap junction (GJ) channels in the proliferation and migration of VSMCs, as well as their contribution to RS still requires further investigation.

GJs are direct intercellular communication pathways, which consist of assemblies of channel proteins known as connexins (9). Ions, second messengers and small metabolites of up to $1 \mathrm{kDa}$ in molecular mass may be rapidly exchanged through GJs (10). However, the precise assembly of their component connexins influences the properties of GJ channels, including permeability and conductance (11). In arteries, the endothelium expresses three connexin isotypes, connexin (Cx)40, Cx37 and Cx43. By contrast, VSMCs predominantly express Cx43 and, in some instances, $\mathrm{Cx} 40$ or $\mathrm{Cx} 45$ (12-14). It has been reported that the expression of $\mathrm{Cx} 43$ is upregulated during the alteration of the VSMC phenotype (15). Moreover, Cx43 remodeling involving changes in size, structure, quantity and distribution may also occur in vascular lesions (16). Cx43 remodeling influences not only the conductivity and permeability of the GJ itself, but also the electrical, chemical and metabolic channels between adjacent cells (17-19). In our previous study, we found that Cx43 was involved in the development of vascular RS following angioplasty-induced balloon injury (20). However, the underlying mechanisms remain unclear.

In the present study, we futher examined whether the knockdown of $\mathrm{Cx} 43$ attenuates vascular RS following angioplasty-induced balloon injury through the inhibition of VSMC proliferation and migration. Cx43-RNAi-LV, a lentiviral vector expressing shRNA targeting Cx43, was used to silence the mRNA and protein expression of Cx43 in the VSMCs. The results of 3-(4,5-dimethylthiazol-2yl-)-2,5-diphenyl tetrazolium bromide (MTT) and Transwell assays revealed that the knockdown of Cx43 by Cx43-RNAi-LV significantly inhibited the proliferation and migration of VSMCs in vitro. In addition, the knockdown of Cx43 effectively attenuated the development of vascular RS and intimal hyperplasia following balloon injury in vivo. Our data further indicate that $\mathrm{Cx} 43$ plays a role in the development of vascular RS and intimal hyperplasia by regulating the proliferation and migration of VSMCs.

\section{Materials and methods}

Experimental animals. Male Sprague-Dawley rats (purchased from the Department of Animal Science, Nanchang University, Nanchang, China) weighing 300-400 g were maintained on a regular chow diet prior to the study. All animal experimental procedures were in accordance with the current National Institutes of Health Guidelines, and they were approved by the Ethics Committee for Animal Experiments at Nanchang University.

Cell culture. The culture of the VMSCs was performed as previously described (21). Briefly, the rats were anesthetized with an intraperitoneal injection of Hydral $(10 \%, 3.5 \mathrm{ml} / \mathrm{kg}$; Harbin Pharmaceutical Group Co., Ltd., Harbin, China) and the carotid artery was quickly removed under aseptic conditions. After stripping the extravascular connective tissue, the vessels were longitudinally cut off and the internal membrane was positioned upward in a glass culture dish. Endothelial denudation was achieved by digestion with $0.25 \%$ trypsin. The tunica media vasorum was cut into $1-\mathrm{mm}^{2}$-sized tissue blocks. The internal membrane was positioned upward in a plastic culture dish. These tissue blocks were incubated at $37^{\circ} \mathrm{C}$ in a $5 \% \mathrm{CO}_{2}$ saturated humidity incubator for $1-2 \mathrm{~h}$ and submerged in $2 \mathrm{ml}$ medium after firm adhesion. After 5-7 days, the VSMCs had migrated from the tissue blocks in Dulbecco's modified Eagle's medium (DMEM) supplemented with $10 \%$ fetal calf serum (Trans Serum ${ }^{\mathrm{TM}}$; Transgen Biotech, Beijing, China) and $1 \%$ penicillin/streptomycin (P/S; Solarbio, Beijing, China). Third to fifth generation VSMCs were used in the experiments.

Construction of GFP-Cx43-shRNA-lentiviral vectors. To examine the role of $\mathrm{Cx} 43$ in vascular $\mathrm{RS}$, a lentiviral vector expressing shRNA targeting Cx43 (Cx43-RNAi-LV) was constructed (GeneChem, Shanghai, China). Briefly, the shRNA sequence for Cx43 (5'-AGAGCACGGCAAGGTGAAA-3') was designed using the manufacturer's RNA interference (RNAi) designer program, and the negative control construct (control shRNA) was created using a scrambled sequence (5'-TTCTCCGAACGTGTCACGT-3'), as described in a previous study (22). DNA oligos were chemically synthesized (GeneChem), annealed and inserted into the expression vector by double digestion with AgeI and EcoRI (New England Biolabs, Ipswich, MA, USA), and ligated with T4 DNA ligase (Takara, Dalian, China) in accordance with the manufacturer's instructions. The ligation was transformed into competent E. coli cells and confirmed by restriction enzyme analysis and DNA sequencing. The sequences were then cloned into pGCSIL-GFP to generate lentiviral vectors. The expression vectors and package vectors were transfected into 293T cells (ATCC, Manassas, VA, USA) using Lipofectamine 2000 (Invitrogen, Carlsbad, CA, USA). After $48 \mathrm{~h}$ of culture, the supernatants containing lentiviruses, such as Cx43-RNAi-LV and NC-GFP-LV (negative control) were harvestedy. 
Purification was then performed using ultracentrifugation and the lentiviral titer was calculated (as $10^{7} \mathrm{TU} / \mu \mathrm{l}$ ).

Reverse transcription-polymerase chain reaction ( $R T-P C R)$. Total RNA was extracted from the rat carotid tissue using TRIzol reagent (Tiangen, Beijing, China), and cDNA was synthesized from the extracted total RNA using the SuperScript III kit (Promega, Madison, WI, USA) following the manufacturer's instructions. The specific primers for PCR were as follows: Cx43 sense, 5'-AAAGGCGTTAAGGAT CGCGTG-3' and antisense, 5'-GTCATCAGGCCGAGG CCT-3' (23); $\beta$-actin sense, 5'-CCCATCTATGAGGGTT ACGC-3' and antisense, 5'-TTTAATGTCACGCACGAT TTC-3'. All specific primers were chemically synthesized (Generay Biotech Co. Ltd., Shanghai, China). The PCR reactions were performed using the GeneAmp PCR System 9700 (Applied Biosystems, Foster City, CA, USA). The amplification conditions for $\mathrm{Cx} 43$ were as follows: $94^{\circ} \mathrm{C}$ for $2 \mathrm{~min}$ followed by 32 cycles at $94^{\circ} \mathrm{C}$ for $45 \mathrm{sec}, 58^{\circ} \mathrm{C}$ for $45 \mathrm{sec}$, and $72^{\circ} \mathrm{C}$ for $90 \mathrm{sec}$, and the final extension at $72^{\circ} \mathrm{C}$ for $5 \mathrm{~min}$. The PCR conditions for $\beta$-actin were as follows: $94^{\circ} \mathrm{C}$ for $2 \mathrm{~min}$ followed by 28 cycles at $94^{\circ} \mathrm{C}$ for $30 \mathrm{sec}, 56^{\circ} \mathrm{C}$ for $30 \mathrm{sec}$, and $72^{\circ} \mathrm{C}$ for $90 \mathrm{sec}$, and the final extension at $72^{\circ} \mathrm{C}$ for $5 \mathrm{~min}$. The amplified RT-PCR products were separated on $1.2 \%(\mathrm{w} / \mathrm{v})$ agarose containing ethidium bromide (both from Sangon Biotech, Shanghai, China) for $30 \mathrm{~min}$. The results of electrophoresis were photographed using the Molecular Imager ${ }^{\circledR}$ ChemiDoc $^{\mathrm{TM}} \mathrm{XRS}+$ system, and the signal densities of the gels were analyzed by Quantity One software (both from Bio-Rad, Hercules, CA, USA).

Western blot analysis. The VSMCs were harvested and treated with radioimmunoprecipitation assay (RIPA) lysis buffer (Sangon Biotech). The whole cell lysate was resuspended in sample buffer containing 4\% sodium dodecyl sulfate (SDS; Sangon Biotech). Proteins were separated by SDS-polyacrylamide gel electrophoresis (PAGE) and transferred onto nitrocellulose membranes (Millipore, Bedford, MA, USA). The membranes were blocked in 5\% skim milk for $1 \mathrm{~h}$ at room temperature, and incubated with rabbit polyclonal anti-Cx43 antibody (71-0700; 1:250 in 5\% skim milk; Zymed Laboratories, San Francisco, CA, USA) or rabbit monoclonal anti-GAPDH antibody (ABS16; 1:1,000 in $5 \%$ skim milk; Chemicon, Temecula, CA, USA) overnight at $4^{\circ} \mathrm{C}$. The membranes were then washed 3 times with TBST, and then incubated with the relative HRP-conjugated IgG secondary antibody (BV-S8008; Zhongshan Golden Bridge Biotechnology Co., Beijing, China) for $2 \mathrm{~h}$ at room temperature, and washed in TBST 3 times. A chemiluminescence assay was carried out with Amersham ECL Prime Western Blotting Detection reagents, and the immunoblotting signal was detected using the Molecular Imager ${ }^{\circledR}$ ChemiDoc $^{\mathrm{TM}}$ XRS+ system (Bio-Rad). The intensity of each $\mathrm{Cx} 43$ band was normalized to the GAPDH band, and the relative expression of Cx43 following viral infection was normalized to the control.

MTT assay. MTT assay was applied to assess the proliferation of the VSMCs according to the manufacturer's instructions. Briefly, $5 \times 10^{4}$ cells were seeded in a 96-well tissue culture plates and cultured in growth medium. The VSMCs were infected with NC-GFP-LV or Cx43-RNAi-LV at an MOI 0, 50, 100, 150 and 200. At 24, 48, 72 and $96 \mathrm{~h}$ after viral infection, $20 \mu \mathrm{l}$ of the MTT solution were added to each well $(5 \mathrm{mg} / \mathrm{ml}$, $0.5 \% \mathrm{MTT}$ ) and the cells were cultured for a further $4 \mathrm{~h}$ to detect the proliferation of the VSMCs. Subsequently, $100 \mu 1$ of dimethyl sulfoxide were added to each well, and the culture plate was shaken at a low speed for 10 min until the crystals dissolved completely. The color intensity was measured spectrophotometrically using a microplate reader (Multiskan FC; Thermo Fisher Scientific, Waltham, MA, USA) at $492 \mathrm{~nm}$. All assays were performed at least 3 times.

Transwell assay. Transwell assays were performed with Transwell chambers (Corning Inc., Corning, NY, USA) as previously described (24). Briefly, 5.0 $\times 10^{4}$ VSMCs were seeded into the upper chamber with $200 \mu \mathrm{l}$ of serum-free medium following infection with NC-GFP-LV or Cx43-RNAi-LV at an MOI of 100. The bottom of the upper chamber was incubated in $500 \mu \mathrm{l}$ of complete medium containing $10 \%$ fetal calf serum and $1 \% \mathrm{P} / \mathrm{S}$. Following $12 \mathrm{~h}$ of incubation, the cells on the top surface of the insert were gently removed with a cotton swab. The migrated cells on the lower surface were fixed with 4\% paraformaldehyde and stained with crystal violet (both from Sigma-Aldrich, St. Louis, MO, USA) for $30 \mathrm{~min}$. The migrated cells were photographed and counted in 4 random fields.

Establishment of model of vascular RS induced by balloon injury. The rats were anesthetized with an intraperitoneal injection of Hydral $(10 \%, 3.5 \mathrm{ml} / \mathrm{kg}$; Harbin Pharmaceutical Group Co., Ltd.). To establish a model of vascular RS, the angioplasty balloon $(1.5 \times 20 \mathrm{~mm}$; Cordis Corp., Miami, FL, USA) was inserted into the rat common carotid artery through an incision in the left external carotid artery as previously described (25). The balloon was then inflated sufficiently in the carotid artery and was drawn 3 times consistently from proximal area to the carotid bifurcation to produce endothelial denudation. The external carotid was ligated and blood flow in the common carotid was restored. To examine the effects of Cx43-RNAi-LV on balloon injury-induced vascular RS, the rats were randomly divided into 3 groups ( $n=6 /$ group): control group (no balloon injury); injury group (balloon injury only) and Cx43-RNAi-LV-treated injury group (balloon injury + Cx43-RNAi-LV). The lentivirus $\left(5 \times 10^{8} \mathrm{TU} / \mathrm{ml}, 100 \mu \mathrm{l}\right)$ expressing shRNA was injected into the balloon-injured rat carotid arteries using a polyethylene catheter, to knockdown Cx43 in the VSMCs. Benzylpenicillin sodium (40x10 $\mathrm{IU} /$ day for 3 days; Harbin Pharmaceutical Group Co., Ltd.,) was then administered by intramuscular injection to prevent infection. All the rats were euthanized by an overdose of Hydral at 28 days following balloon injury. Three serial cryosections ( $5 \mu \mathrm{m}$ thick) were prepared from the middle portion of the rat common carotid arteries. The slices were stained with hematoxylin and eosin (H\&E; Sangon Biotech, Shanghai, China), and histomorphologic observation was performed under a light microscope (Olympus, Tokyo, Japan) to examine the structure of the blood vessel wall following balloon injury. The intimal and medial area of 3 serial cryosections from each sample was measured using Image-Pro Plus 5.0 software (Media Cybernetics, Inc., Houston, TX, USA) in order to evaluate vascular remodeling. 


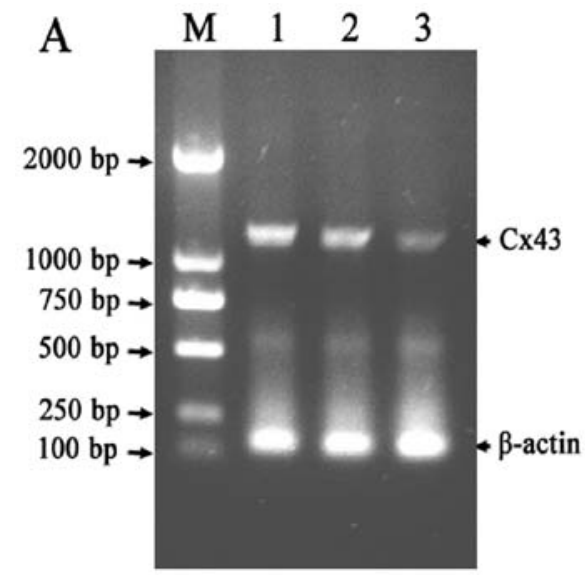

B

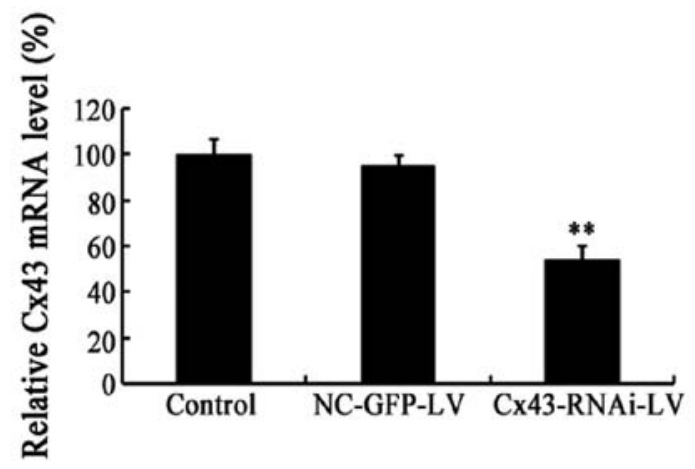

Figure 1. Silencing the mRNA expression of connexin 43 (Cx43) in cultured vascular smooth muscle cells (VSMCs) using Cx43-RNAi-LV. (A) Electrophoresis of RT-PCR products from Cx43 and $\beta$-actin on an agarose gel. Lane M, DNA ladder (2,000, 1,000, 750, 500, 250 and 100 bp from top to bottom, respectively); lane 1, control VSMCs; lane 2, NC-GFP-LV-infected VSMCs, lane 3, Cx43-RNAi-LV-infected VSMCs. $\beta$-actin was used as an endogenous control (B) Quantification of the relative Cx43 mRNA level in each group. The relative Cx43 mRNA level is indicated as the normalization of the ratio of Cx43/ $\beta$-actin in each sample to the control. Data represent the means \pm SD of at least 3 independent experiments. ${ }^{* *} \mathrm{P}<0.01$ vs. controls (uninfected cells).

A

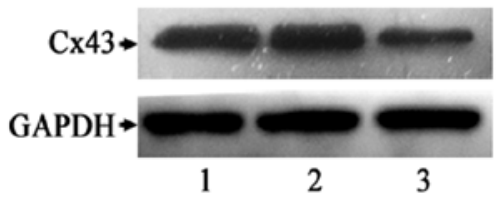

B

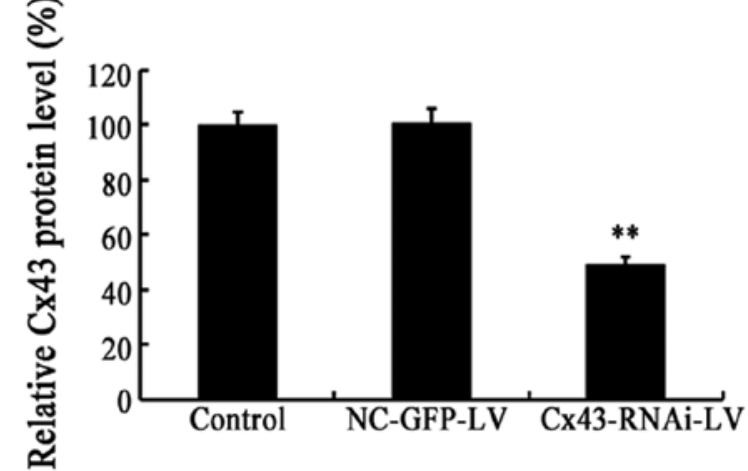

Figure 2. Knockdown of connexin 43 (Cx43) protein expression in cultured vascular smooth muscle cells (VSMCs) by Cx43-RNAi-LV. (A) After infection of the lentiviral vectors, NC-GFP-LV or Cx43-RNAi-LV, the VSMCs were harvested and the protein expression of Cx43 in each group was examined by western blot analysis. GAPDH was used as an endogenous control. Lane 1, control VSMCs; lane 2, NC-GFP-LV-infected VSMCs, lane 3, Cx43-RNAi-LV-infected VSMCs. (B) Quantification of relative Cx43 expression in each group. The relative Cx43 protein level is indicated as the normalization of the ratio of Cx43/GAPDH in each sample to the control. Data represent the means \pm SD of at least 3 independent experiments. ${ }^{* *} \mathrm{P}<0.01$ vs. control (uninfected cells).

Statistical analysis. The quantitative data are presented as the means \pm standard deviation (SD). Data were analyzed using the Student's t-test to compare two conditions, and a value of $\mathrm{P}<0.05$ was considered to indicate a statistically significant difference.

\section{Results}

Lentivirus-mediated knockdown of Cx43 in cultured VSMCs. In our previous study, it was found that $\mathrm{Cx} 43$ is involved in the development of vascular RS and intimal hyperplasia after balloon injury (20). As the intimal hyperplasia and vascular RS which occur following balloon injury are mainly induced by the proliferation and migration of VSMCs, we used a lentiviral vector expressing shRNA targeting Cx43 (Cx43-RNAi-LV) to silence Cx43 in the VSMCs. The results of RT-PCR revealed that the mRNA level of $\mathrm{Cx} 43$ was significantly decreased in the VSMCs infected with Cx43-RNAi-LV, but not in the control (uninfected) or the NC-GFP-LV-infected cells. Compared with the controls, the mRNA level of $\mathrm{Cx} 43$ in the Cx43-RNAi-LV-infected VSMCs decreased to approximately
54.3\% (Fig. 1A). Moreover, the protein expression of Cx43 in the VSMCs was examined by western blot analysis. Compared with the controls, the protein level of Cx43 in the VSMCs infected with Cx43-RNAi-LV decreased to approximately $49.4 \%$ (Fig. 2). These results indicated that the lentivirus-mediated knockdown of Cx43 in the cultured VSMCs was achieved by infection Cx43-RNAi-LV.

Effect of knockdown of Cx43 on the proliferation of cultured VSMCs. Intimal hyperplasia and vascular RS which occur following balloon injury are mainly induced by the proliferation and migration of VSMCs, and the principal gap junction protein in VSMCs, Cx43, plays an important role in the development of intimal hyperplasia and vascular RS (4). Thus, to examine the role of $\mathrm{Cx} 43$ in the proliferation of VSMCs, Cx43RNAi-LV was used to specifically silence Cx43 in the VSMCs, and the lentiviral vector, NC-GFP-LV, was used as the negative control. The proliferation of the VSMCs under each condition was evaluated by MTT assay. Infection with Cx43-RNAi-LV at an MOI of 100, 150 and 200 significantly inhibited the proliferation of the VSMCs at 24, 48, 72 and $96 \mathrm{~h}$ following 

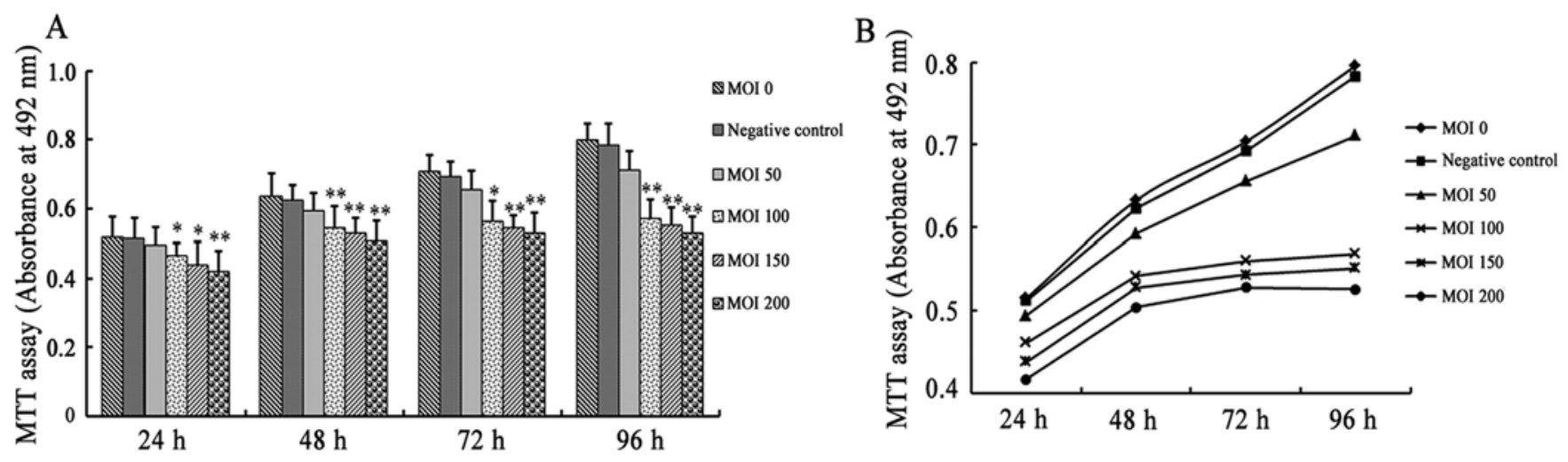

Figure 3. Knockdown of connexin 43 (Cx43) by Cx43-RNAi-LV effectively inhibits the proliferation of vascular smooth muscle cells (VSMCs). (A) Dose-dependent effect of Cx43-RNAi-LV on the proliferation of VSMCs. VSMCs were infected with Cx43-RNAi-LV at various MOIs or with NC-GFP-LV (negative control), and MTT assay was performed at $24,48,72$ and $96 \mathrm{~h}$ following infection. $\mathrm{n}=5$. ${ }^{*} \mathrm{P}<0.05 \mathrm{vs}$. MOI of $0 ;{ }^{* * *} \mathrm{P}<0.01 \mathrm{vs}$. MOI of 0 . (B) Time course of VSMC proliferation following infection with Cx43-RNAi-LV. Following infection with Cx43-RNAi-LV at various MOIs or with NC-GFP-LV (negative control), cell proliferation was assessed by MTT assay every $24 \mathrm{~h}$.
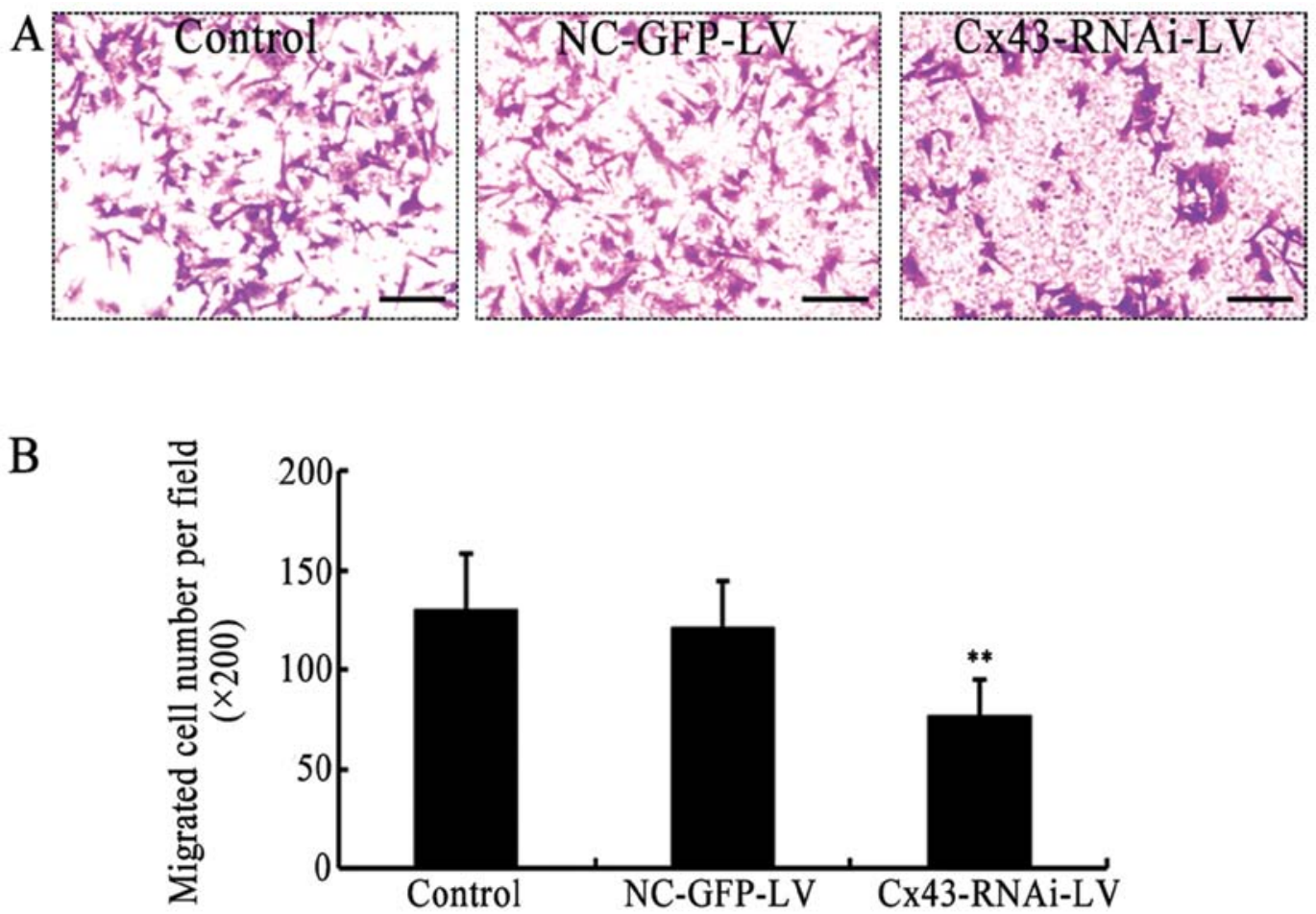

Figure 4. Knockdown of connexin 43 (Cx43) decreases the migratory activity of vascular smooth muscle cells (VSMCs). (A) VSMCs were infected with NC-GFP-LV or Cx43-RNAi-LV at MOI 100, and the migratory activity of VSMCs was evaluated by Transwell assay. The migrated cells under each experimental condition were stained with crystal violet, and they were visualized under a microscope. Scale bar, $100 \mu \mathrm{m}$. (B) The migratory activity of VSMCs under each experimental condition was quantified by the mean migrated cell counts from at least 9 fields in 3 independent experiments. ${ }^{* *} \mathrm{P}<0.01$ vs. control (uninfected cells).

infection (Fig. 3). By contrast, infection with NC-GFP-LV and Cx43-RNAi-LV at an MOI of 50 had no significant effect on the proliferation of the VSMCs. The results of MTT assay indicated that the gap junction protein, $\mathrm{Cx} 43$, is important for the proliferation of VSMCs.

Knockdown of CX43 effectively inhibits the migratory activity of the VSMCs. In addition, the migration of the VSMCs is involved in the development of intimal hyperplasia and vascular RS (4). Thus, to examine the role of Cx43 in the migra- tion of VSMCs, Cx43 in the VSMCs was specifically silenced by Cx43-RNAi-LV. The migratory activity of the VSMCs in which $\mathrm{Cx} 43$ was knocked down or not was evaluated by Transwell assay. Compared with the control (uninfected) or the negative control (NC-GFP-LV), the knockdown of Cx43 by Cx43-RNAi-LV significantly attenuated the migration of the VSMCs (Fig. 4). Taken together, the results of MTT assay and Transwell assay, provide direct evidence that $\mathrm{Cx} 43$ is a vital regulatory factor in the proliferation and migration of VSMCs. Our results also suggest that $\mathrm{Cx} 43$ is involved in the develop- 

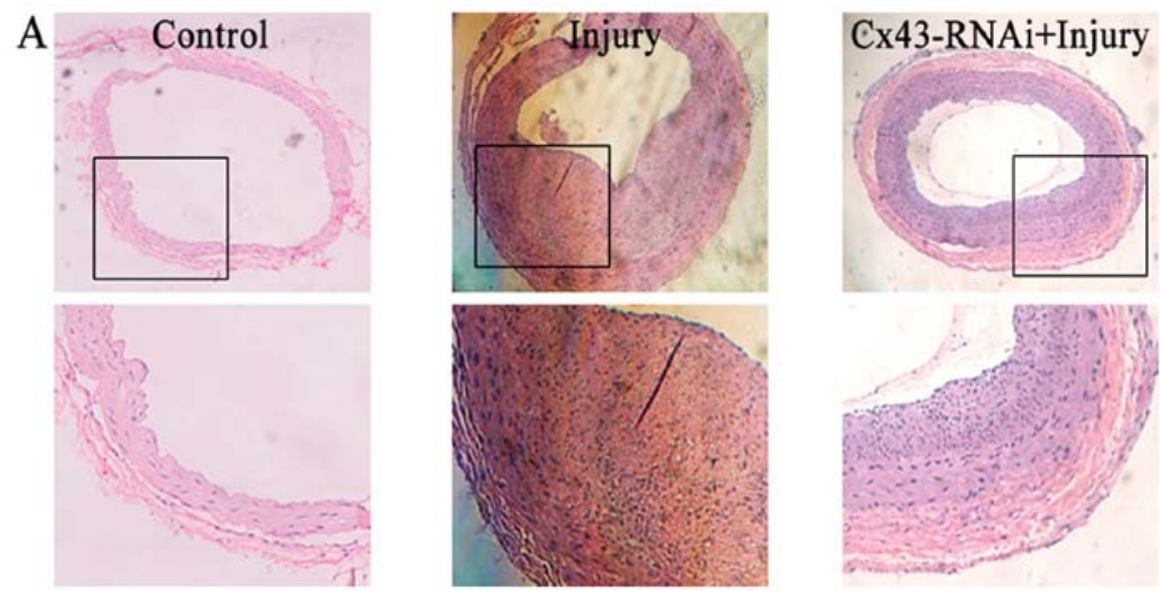

B

$\square$ medial area $\quad$ o intimal area

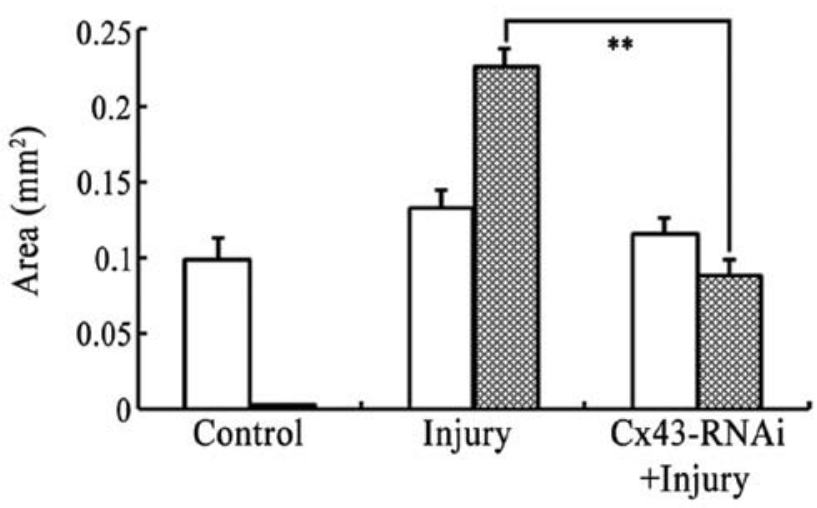

Figure 5. Knockdown of connexin 43 (Cx43) attenuates the development of intimal hyperplasia and restenosis (RS) induced by balloon injury. (A) Histomorphological observations of the arteries. Carotid arteries were removed after the indicated treatments and they were cut into sections (5- $\mu \mathrm{m}$-thick) for hematoxylin and eosin (H\&E) staining and subsequent examination under a microscope. Images at low and high magnification are shown in the upper and lower panels, respectively (upper panel, x100 magnification; bottom panel, x400 magnification). The normal artery without balloon injury is shown in the left panel (control). The artery after balloon injury is shown in the middle panel. The artery treated with Cx43-RNAi after balloon injury is shown in the right panel. (B) The intimal and medial area of the arterial sections was measured using Image-Pro Plus 5.0 software. Data represent the means \pm SD of 6 independent experiments. ${ }^{* *} \mathrm{P}<0.01$.

ment of intimal hyperplasia and vascular RS which occur following balloon injury, by regulating the proliferation and migration of VSMCs.

Knockdown of Cx43 attenuates the development of intimal hyperplasia and vascular $R S$ induced by balloon injury in vivo. Since the proliferation and migration of VSMCs is important in the development of intimal hyperplasia and vascular RS following balloon injury, and the knockdown of Cx43 effectively attenuated the proliferation and migration of the VSMCs (Figs. 3 and 4), we further examined whether the knockdown of $\mathrm{Cx} 43$ using Cx43-RNAi-LV prevents the development of intimal hyperplasia and vascular RS following balloon injury in vivo. The histomorphological observations of the arterial sections (Fig. 5A) and statistical analysis of the intimal/medial areas (Fig. 5B) revealed that the lentivirusmediated knockdown of Cx43 by Cx43-RNAi-LV significantly attenutated the development of balloon injury-induced intimal hyperplasia and vascular RS. These results are consistent with the results of MTT assay and Transwell assay in vitro, and suggest that $\mathrm{Cx} 43$ is involved in the development of intimal hyperplasia and vascular RS following balloon injury, through the regulation of the proliferation and migration of VSMCs.

\section{Discussion}

Vascular RS and intimal hyperplasia are the main adverse effects of PCI, which usually limit the clinical effects of PCI. Evidence indicates the vital role of endothelial denudation, as well as the proliferation and migration of VSMCs in vascular RS and intimal hyperplasia $(1,2)$. The related molecular mechanisms, nevertheless, remain unclear. On the other hand, studies have suggested the involvement of GJs in the development of some vascular diseases, such as hypertension, atherosclerosis and RS $(24,26)$. Particularly, the principal GJ protein, Cx43, in VSMCs may be involved in the development of vascular RS and intimal hyperplasia (20,27). In transgenic mice, it was found that a reduced $\mathrm{Cx} 43$ expression effectively inhibited acute neointimal formation in hypercholesterolemic mice (28). In our previous study (20), we also found that the expression of the GJ protein, Cx43, was upregulated in vessels following balloon injury. The knockdown of $\mathrm{Cx} 43$ effectively prevented 
balloon injury-induced vascular RS and intimal hyperplasia (20). Moreover, evidence suggests that GJs are involved in the proliferation and migration-related signaling pathways of VSMCs $(22,29)$. The blockade of Cx43 hemichannels has been shown to reduce neointima formation by inhibiting the proliferation and phenotypic modulation of VSMCs (30). Cx43 is also involved in the angiotensin II- or oxidized-phospholipidinduced migration and proliferation of VSMCs $(8,31,32)$. However, direct evidence is still lacking as to the role of $\mathrm{Cx} 43$ in the proliferation and migration of VSMCs induced by endothelial denudation or balloon injury. Therefore, it is interesting to investigate the direct effects of $\mathrm{Cx} 43$ silencing on the proliferation and migration of VSMCs and the development of vascular RS following PCI.

In the present study, we constructed a lentiviral vector expressing shRNA targeting Cx43, Cx43-RNAi-LV, to silence Cx43. Cx43-RNAi-LV effectively knocked down Cx43 in the cultured VSMCs (Figs. 1 and 2). These results are consistent with the in vivo results of our previous study (20). To examine the effects of Cx43-RNAi-LV on the proliferation of VSMCs, the cells were infected with $\mathrm{Cx} 43-\mathrm{RNAi}-\mathrm{LV}$ at various MOIs ( 0 , 50, 100, 150 and 200). Compared with infection at an MOIof 0 or infection with the negative control, infection with $\mathrm{Cx} 43$ RNAi-LV at MOIs of 100, 150 and 200 significantly inhibited the proliferation of VSMCs (Fig. 3). In addition, the migration of the cultured VSMCs under each experimental condition was evaluated by Transwell assay. Infection with Cx43-RNAi-LV at an MOI of 100 significantly attenuated the migratory activity of the VSMCs (Fig. 4). By contrast, infection with NC-GFP-LV had no significant effect on the migration of the VSMCs. These in vitro results are consistent with those of a previous study (30), and provide direct evidence that $\mathrm{Cx} 43$ plays an important role in regulating the proliferation and migration of VSMCs. On the other hand, the lentivirus-mediated knockdown of Cx43 effectively attenuated the development of vascular RS and intimal hyperplasia following balloon injury (Fig. 5). Taken together, these results indicate that $\mathrm{Cx} 43$ contributes to the development of vascular RS and intimal hyperplasia through the regulation of the proliferation and migration of VSMCs. Although the detailed mechanisms involved remain unclear, it has been suggested the MAPK signaling pathway regulates the role of Cx43 in the proliferation and migration of VSMCs $(8,33)$. The MAPK-AP-1 signaling pathway has been found to regulate the role of $\mathrm{CX} 43$ in the angiotensin II-induced migration and proliferation of saphenous vein smooth muscle cells (8). Moreover, the MAPK-mediated phosphorylation of $\mathrm{Cx} 43$ promotes the proliferation of VSMCs through the facilitation of the binding of Cx43 to cell cycle protein cyclin E (33).

In conclusion, our data demonstrate that the knockdown of the principal GJ protein in VSMCs, Cx43, may be an effective measure to prevent the development of intimal hyperplasia and vascular RS through the inhibition of the proliferation and migration of VSMCs. In the present study, we constructed a lentiviral vector expressing shRNA targeting Cx43. Infection with $\mathrm{Cx} 43$ RNAi-LV effectively silenced the mRNA and protein expression of $\mathrm{Cx} 43$ in the VSMCs. In addition, the knockdown of Cx43 with Cx43-RNAi-LV significantly inhibited the proliferation and migration of VSMCs in vitro, and effectively attenuated the development of vascular RS and intimal hyperplasia following balloon injury in vivo. These results demonstrate that the GJ protein, $\mathrm{Cx} 43$, plays an important role in the development of vascular RS and intimal hyperplasia through the regulation of the proliferation and migration of VSMCs. Thus, our data suggest that $\mathrm{Cx} 43$ may be a novel and promising pharmacological target for preventing the development of intimal hyperplasia and RS following PCI.

\section{Acknowledgements}

This study was supported by the National Natural Science Foundation of China (grant nos. 30760286 and 81241125), the Jiangxi Province Natural Science Foundation (grant no. 20142BAB205022), and partially by the National Natural Science Foundation of China (grant nos. 31360241 and 81472371), and the Postgraduate Student Foundation for New Teachers from the Ministry of Education of China (no. 20123601120001).

\section{References}

1. Trikalinos TA, Alsheikh-Ali AA, Tatsioni A, Nallamothu BK and Kent DM: Percutaneous coronary interventions for non-acute coronary artery disease: a quantitative 20 -year synopsis and a network meta-analysis. Lancet 373: 911-918, 2009.

2. Meads C, Cummins C, Jolly K, Stevens A, Burls A and Hyde C: Coronary artery stents in the treatment of ischaemic heart disease: a rapid and systematic review. Health Technol Assess 4: $1-153,2000$.

3. Odell A, Grip L and Hallberg LR: Restenosis after percutaneous coronary intervention (PCI): experiences from the patients' perspective. Eur J Cardiovasc Nurs 5: 150-157, 2006.

4. Zhang C, Chaturvedi D, Jaggar L, Magnuson D, Lee JM and Patel TB: Regulation of vascular smooth muscle cell proliferation and migration by human sprouty 2 . Arterioscler Thromb Vasc Biol 25: 533-538, 2005.

5. Owens GK: Regulation of differentiation of vascular smooth muscle cells. Physiol Rev 75: 487-517, 1995.

6. Crowley ST, Ray CJ, Nawaz D, Majack RA and Horwitz LD: Multiple growth factors are released from mechanically injured vascular smooth muscle cells. Am J Physiol 269: H1641-H1647, 1995.

7. Li F,Zhang C, Schaefer S, Estes A and Malik KU: ANG II-induced neointimal growth is mediated via cPLA2- and PLD2-activated Akt in balloon-injured rat carotid artery. Am J Physiol Heart Circ Physiol 289: H2592-H2601, 2005.

8. Jia G, Cheng G, Gangahar DM and Agrawal DK: Involvement of connexin 43 in angiotensin II-induced migration and proliferation of saphenous vein smooth muscle cells via the MAPK-AP-1 signaling pathway. J Mol Cell Cardiol 44: 882-890, 2008.

9. Hervé JC, Phelan P, Bruzzone R and White TW: Connexins, innexins and pannexins: bridging the communication gap. Biochim Biophys Acta 1719: 3-5, 2005.

10. Bruzzone R, White TW and Paul DL: Connections with connexins: the molecular basis of direct intercellular signaling. Eur J Biochem 238: 1-27, 1996.

11. Elfgang C, Eckert R, Lichtenberg-Fraté H, Butterweck A, Traub O, Klein RA, Hülser DF and Willecke K: Specific permeability and selective formation of gap junction channels in connexin-transfected HeLa cells. J Cell Biol 129: 805-817, 1995.

12. van Kempen MJ and Jongsma HJ: Distribution of connexin37, connexin40 and connexin 43 in the aorta and coronary artery of several mammals. Histochem Cell Biol 112: 479-486, 1999.

13. Hong T and Hill CE: Restricted expression of the gap junctional protein connexin 43 in the arterial system of the rat. J Anat 192: 583-593, 1998.

14. Li X and Simard JM: Increase in $\mathrm{Cx} 45$ gap junction channels in cerebral smooth muscle cells from SHR. Hypertension 40: 940-946, 2002.

15. Matsushita T, Rama A, Charolidi N, Dupont E and Severs NJ: Relationship of connexin43 expression to phenotypic modulation in cultured human aortic smooth muscle cells. Eur J Cell Biol 86: 617-628, 2007. 
16. Ram R, Wescott AP, Varandas K, Dirksen RT and Blaxall BC: Mena associates with Rac1 and modulates connexin 43 remodeling in cardiomyocytes. Am J Physiol Heart Circ Physiol 306: H154-H159, 2014

17. Kieken F, Mutsaers N, Dolmatova E, Virgil K, Wit AL, Kellezi A, Hirst-Jensen BJ, Duffy HS and Sorgen PL: Structural and molecular mechanisms of gap junction remodeling in epicardial border zone myocytes following myocardial infarction. Circ Res 104: 1103-1112, 2009.

18. Qu J, Volpicelli FM, Garcia LI, Sandeep N, Zhang J, Márquez-Rosado L, Lampe PD and Fishman GI: Gap junction remodeling and spironolactone-dependent reverse remodeling in the hypertrophied heart. Circ Res 104: 365-371, 2009.

19. Rucker-Martin C, Milliez P, Tan S, Decrouy X, Recouvreur M, Vranckx R, Delcayre C, Renaud JF, Dunia I, Segretain D, Hatem SN: Chronic hemodynamic overload of the atria is an important factor for gap junction remodeling in human and rat hearts. Cardiovasc Res 72: 69-79, 2006.

20. Han XJ, Chen M, Hong T, Zhu LY, He D, Feng JG and Jiang LP: Lentivivirus-mediated RNAi knockdown of the gap junction protein, Cx43, attenuates the development of vascular restenosis following balloon injury. Int J Mol Med 35: 885-892, 2015.

21. Zhang J, Guo C, Wang R, Huang L, Liang W, Liu R and Sun B: An Egr-1-specific DNAzyme regulates Egr-1 and proliferating cell nuclear antigen expression in rat vascular smooth muscle cells. Exp Ther Med 5: 1371-1374, 2013.

22. Ai Z, Yin L, Zhou X, Zhu Y, Zhu D, Yu Y and Feng Y: Inhibition of survivin reduces cell proliferation and induces apoptosis in human endometrial cancer. Cancer 107: 746-756, 2006

23. Barac YD, Zeevi-Levin N, Yaniv G, Reiter I, Milman F, Shilkrut M, Coleman R, Abassi Z and Binah O: The 1,4,5-inositol trisphosphate pathway is a key component in Fas-mediated hypertrophy in neonatal rat ventricular myocytes. Cardiovasc Res 68: 75-86, 2005

24. Severs NJ, Rothery S, Dupont E, Coppen SR, Yeh HI, Ko YS Matsushita T, Kaba R and Halliday D: Immunocytochemical analysis of connexin expression in the healthy and diseased cardiovascular system. Microsc Res Tech 52: 301-322, 2001.

25. Meng QH, Yang G, Yang W, Jiang B, Wu L and Wang R: Protective effect of hydrogen sulfide on balloon injury-induced neointima hyperplasia in rat carotid arteries. Am J Pathol 170: 1406-1414, 2007.
26. Brisset AC, Isakson BE and Kwak BR: Connexins in vascular physiology and pathology. Antioxid Redox Signal 11: 267-282, 2009.

27. Déglise S, Martin D, Probst H, Saucy F, Hayoz D, Waeber G, Nicod P, Ris HB, Corpataux JM and Haefliger JA: Increased connexin43 expression in human saphenous veins in culture is associated with intimal hyperplasia. J Vasc Surg 41: 1043-1052, 2005.

28. Chadjichristos CE, Matter CM, Roth I, Sutter E, Pelli G, Lüscher TF, Chanson M and Kwak BR: Reduced connexin43 expression limits neointima formation after balloon distension injury in hypercholesterolemic mice. Circulation 113: 2835-2843, 2006.

29. Chadjichristos CE, Morel S, Derouette JP, Sutter E, Roth I, Brisset AC, Bochaton-Piallat ML and Kwak BR: Targeting connexin 43 prevents platelet-derived growth factor-BB-induced phenotypic change in porcine coronary artery smooth muscle cells. Circ Res 102: 653-660, 2008.

30. Song M, Yu X, Cui X, Zhu G, Zhao G, Chen J and Huang L: Blockade of connexin 43 hemichannels reduces neointima formation after vascular injury by inhibiting proliferation and phenotypic modulation of smooth muscle cells. Exp Biol Med (Maywood) 234: 1192-1200, 2009.

31. Shi Y, Hou X, Zhang X, Wang Y, Chen Y and Zou J: Inhibition of oxidized-phospholipid-induced vascular smooth muscle cell proliferation by resveratrol is associated with reducing $\mathrm{Cx} 43$ phosphorylation. J Agric Food Chem 61: 10534-10541, 2013.

32. Johnstone SR, Ross J, Rizzo MJ, Straub AC, Lampe PD, Leitinger $\mathrm{N}$ and Isakson BE: Oxidized phospholipid species promote in vivo differential cx43 phosphorylation and vascular smooth muscle cell proliferation. Am J Pathol 175: 916-924, 2009.

33. Johnstone SR, Kroncke BM, Straub AC, Best AK, Dunn CA Mitchell LA, Peskova Y, Nakamoto RK, Koval M, Lo CW, et al: MAPK phosphorylation of connexin 43 promotes binding of cyclin E and smooth muscle cell proliferation. Circ Res 111: 201-211, 2012. 